\title{
Photochromic Resins for Secondary Printing in Additive Manufacturing
}

\author{
Fei WANG and Fu-Ke WANG*
}

Polymeric Materials Department, Institute of Materials Research and Engineering, Agency for Science, Technology and Research (A*STAR), 2 Fusionopolis Way, \#08-03 Innovis, Singapore

wangf@imre.a-star.edu.sg

${ }^{*}$ FK Wang

Keywords: Photochromic, 3D printing, additive manufacturing, polymers, resins.

\begin{abstract}
D printing has been identified as the main technique for the coming Industry 4.0 due to its cyber-physical systems and the ability to create complicated 3D object in a fast yet accurate manner. However, the polymers-based 3D printings are currently mainly used for prototype or mold printing due to the lack of functional polymer resins. Functional 3D printing has been one of the most important goal of scientific research and industry development. In the present work, we developed the 3D printable resin which allows the printing of 3D structures with photochromic properties, that is, their color can change reversibly with different light irradiation. By using the reversible photochromic properties, we developed the secondary printing technology that allows the printing of multicolor and different color patterns on the as-printed 3D structures. This will be of great importance and indeed for applications in jewelry design, UV indicator, optically modulated sensors, smart windows, display, package indicators, and so on.
\end{abstract}

\section{Introduction}

3D printing, also known as additive manufacturing, refers to the technology used for rapid production of 3D objects directly from digital design. With the unique ability to create complicated 3D object in a fast yet accurate manner, 3D printing technology is now widely used not just in industry but in households as well.[1-5] However, the polymers-based 3D printings are currently mainly used for prototype or mold printing due to lack of functional polymer resins. Functional 3D printing has been one of the most important goal of scientific research and industry development. One of the most important attempts is what people called 4D printing, where the fourth dimension is time. This concept was first proposed by Skylar Tibbits from MIT Self-Assembly Lab.[6,7] This shape transformation is realized with the help of hydrophilic materials that expand when submerged in water to introduce the designed shape changes. While shape transformation stands as dominated area for 4D printing, development of 4D printing like material, which have the ability to change properties such as color, mechanical properties, conductivity and etc., will be of great importance and indeed have wide application as smart material.[8-11]

In the present work, we developed the 3D printable resin which allows the printing of 3D structures with photochromic properties, that is, their color can change reversibly with different light irradiation. By using the reversible photochromic properties, we developed the secondary printing technology that allows the printing of multicolor and different color patterns on the as-printed 3D structures. This will be of great importance and indeed for applications in jewelry design, UV indicator, optically modulated sensors, smart windows, display, package indicators, and so on.

\section{Results and Discussion}

Photochromic materials can undergo reversible color change upon exposure to light. Normally, the material changes color upon exposure to UV radiation and fades back to its original color upon exposure to visible light or heat. The most famous application of reversible photochromic material is for ophthalmic lenses. Other applications such as 3D optical data storage, supramolecular switches and solar energy storage have been studied and shown potential usage in the future. Moreover, 
photochromic materials have been widely used in cosmetic, toy, clothing, construction industries when novel and fancy color changing is needed in the product. The development of photochromic 3D printing materials will broaden the application of not only photochromic materials but also current 3D printing technologies. Most importantly, the use of photochromic resin in 3D printing will allow printing of multi-colors structures, and reversible color change properties enable the possibility of secondary printing on the 3D printed structures, opening a new door of advanced printing technology, especially for industry design.

A typical photopolymer resin for SLA/DLP 3D printing consists of polymerizable monomer/oligomer, photoinitiator and photo absorber.[12,13] The role of photo absorber is to control the curing depth to ensure satisfied printing accuracy. We used Di(ethylene glycol) diacrylate (DEGDA) and 1,6-Hexanediol diacrylate (HDDA) with weight ratio of 17:3 as polymerizable components. DEGDA can introduce good mechanical properties into printed object and HDDA can help to improve thesolubility of photoinitiator and photo absorber. Commonly used photoinitiator Phenylbis(2,4,6-trimethylbenzoyl)phosphine oxide was added in $0.5 \mathrm{wt} \%$, which was found to be enough for the printing. For photo absorber, we selected fluorescein as its light color can ensure good visibility of color changing from chromic dye. Based on this, we've prepared three photopolymer resins with different photochromic dyes and selected $1^{\prime}, 3^{\prime}$-Dihydro- 1 ', $3^{\prime}, 3^{\prime}$ -trimethyl-6-nitrospiro[2H-1-benzopyran-2,2 ' $-(2 \mathrm{H})$-indole](0.25 wt\%) as dye for red color changing

IMRE-PC-R), 1,2-Bis(2,4-dimethyl-5-phenyl-3-thienyl)-3,3,4,4,5,5-hexafluoro-1-cyclopentene(0.10 wt\%) as dye for green color changing (Resin: IMRE-PC-G) and $1^{\prime}, 3^{\prime}$-Dihydro-1 ', $3^{\prime}, 3^{\prime}$ -trimethyl-6-nitrospiro[2H-1-benzopyran-2,2 ' -(2H)-indole](0.20 wt\%) as dye for blue color changing (Resin: IMRE-PC-B).
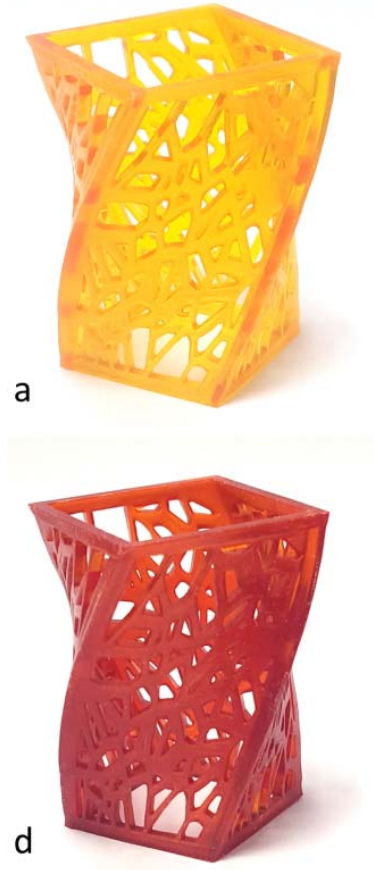

b

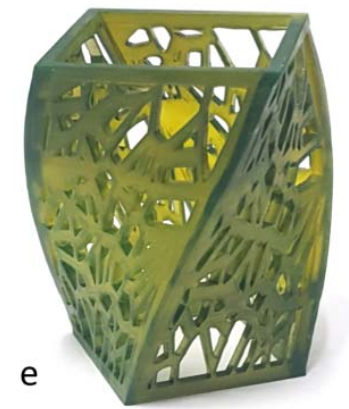

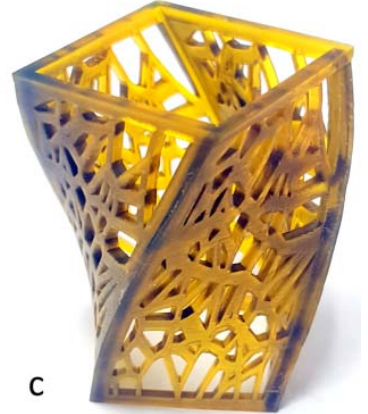

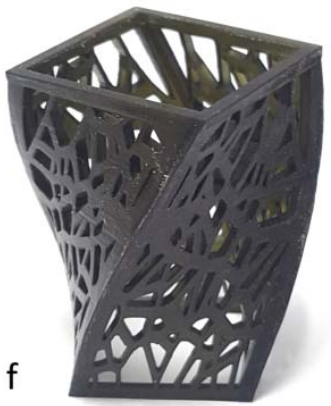

$10 \mathrm{~mm}$

Figure 1. Photos of as printed 3D vases structures using resin IMRE-PC-R (a), IMRE-PC-G (b) and IMRE-PC-B (c) and the corresponding structures after exposure to UV radiation: (d) IMRE-PC-R, (e) IMRE-PC-G and (f) IMRE-PC-B.

Prototypes printing was carried out using and DLP 3D printer (Brand: LittleRP) with a DLP projector as light source (Brand \& model: Acer P1283) and Creation Workshop as printing control software. The printing parameters such as concentration of photoinitiators, photo absorber, chromic 
dyes as well as printing speeds and exposing time for each layer were optimized by using cure depth studies according to our previous report. [14] Fig. 1 displays the as printed 3D vase structures by using resins IMRE-PC-R (a), IMRE-PC-G (b) and IMRE-PC-B (c) with color of orange, yellow and brown, respectively. These printed structures show uniform color and expected 3D geometry as designed, suggesting that these photochromic dyes were homogeneously dispersed in the polymer resins and didn't affect the printing accuracy. Most importantly, upon exposure to UV radiation, these printed objects changed to the design color as shown in Fig. 1. The color of the printed prototypes that printed using resins IMRE-PC-R, IMRE-PC-G and IMRE-PC-B change their colors to red, green and dark blue respectively. In addition, these new colors will returned to their original colors if these printed structures were left under visible light (room light). The reversible color change observed here indicated that the chemical structures of photochromic dyes were not affected by the photo-radical polymerization reaction during printing process.
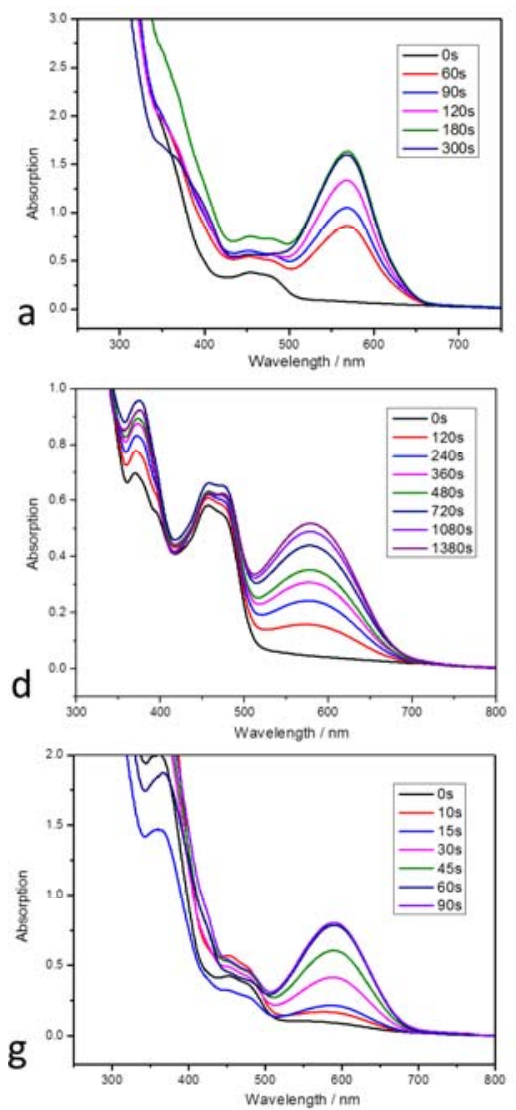
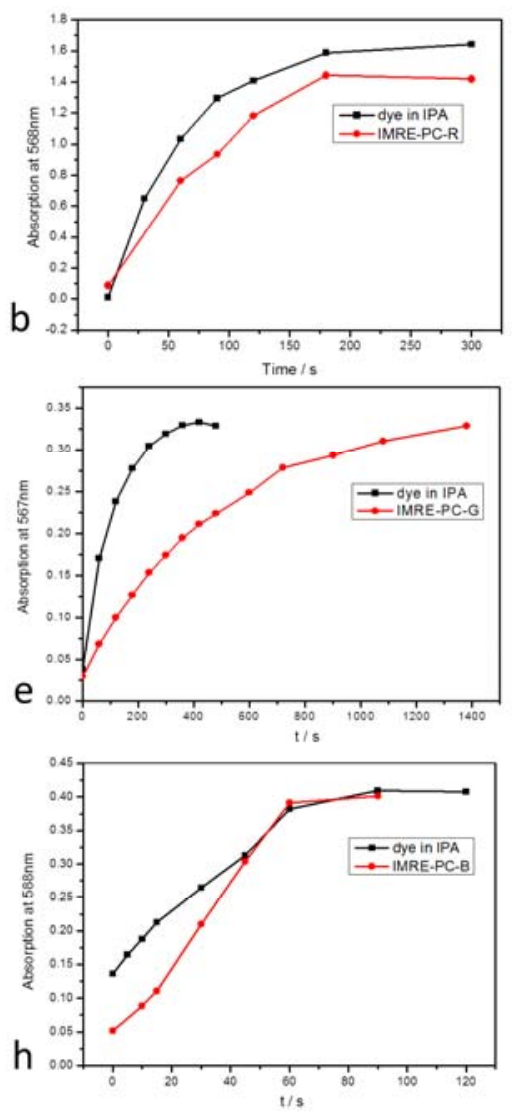
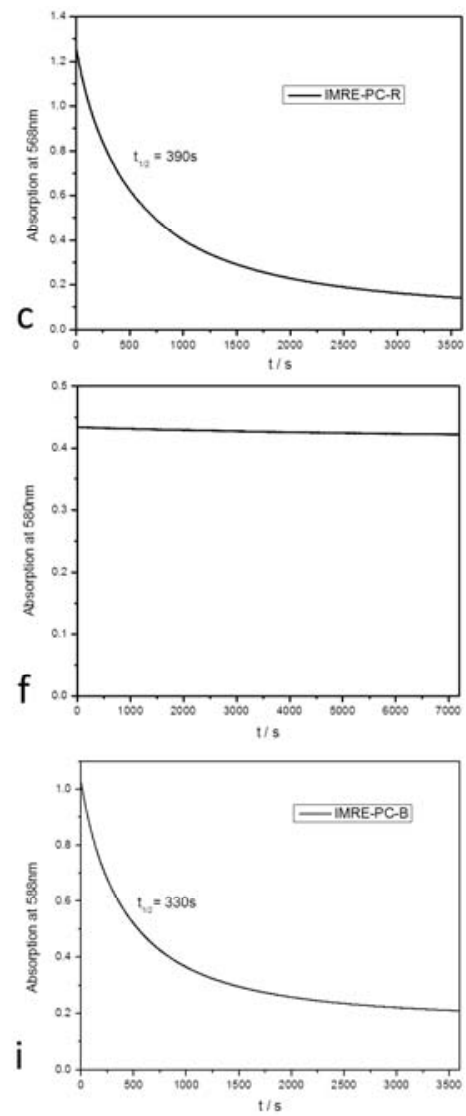

Figure 2. UV-Vis spectrum of printed thin film was measured before and after exposure to UV radiation for a certain time (Figure 2a for IMRE-PC-R, 2d for IMRE-PC-G and 2g IMRE-PC-B); Color changing curves and decoloring curves for thin film printed using resin IMRE-PC-R (2b\& 2c), IMRE-PC-G (2e\& 2f) and IMRE-PC-B (2h\& 2i).

We then investigated the coloring speed for the selected photochromic dyes in the polymer matrix. For this purpose, a thin film with thickness of $0.25 \mathrm{~mm}$ was printed by $3 \mathrm{D}$ printer using our resins. The UV-Vis spectrum of printed thin film was measured before and after exposure to UV radiation for a certain time (Fig. 2a, 2d and 2g). From the UV-Vis spectrums, we can tell that thin film by resin IMRE-PC-B showed the fastest color changing speed and reached the highest conversion at around 60 seconds. On the other hand, thin film printed from resin IMRE-PC-G exhibited the slowest color change speed as the color changing was continue even after explosion to UV radiation for 20 mins. Compared the color changing curve of photochromic dyes in solution and in the printed film, slower color changing speed was observed in 3D printed thin film. This phenomenon can be explained by the rigidity of polymer matrix, which inhibits the photo-induced ring opening reaction of photochromic 
dyes. We also measured the de-coloring curves of printed thin films. Similar with observation of color changing speed, thin film by resin IMRE-PC-B showed the fastest decoloring speed with half-life time $t 1 / 2=330$ s and faded back to its original color with $1 \mathrm{~h}$. In contrast, thin film by resin IMRE-PC-G needs more than $12 \mathrm{~h}$ for complete decoloring (Fig. 2c, 2f \& 2i).

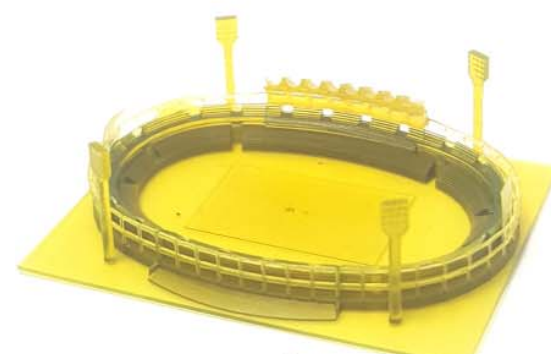

a

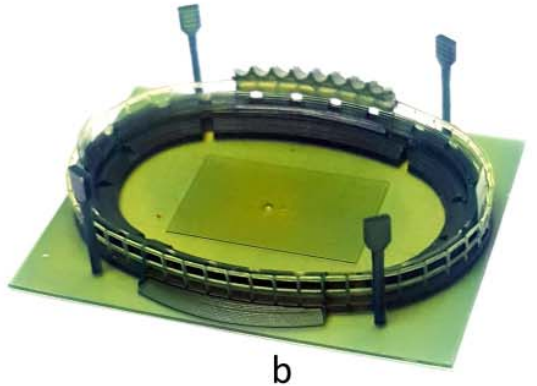

b

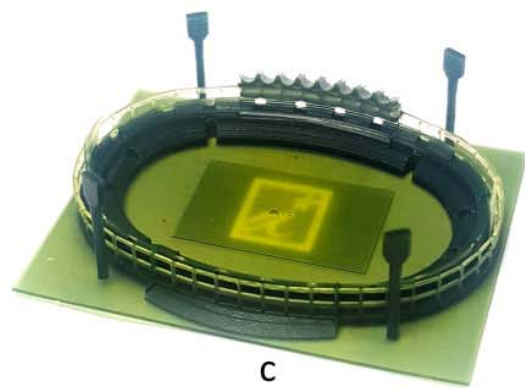

C

Figure 3. Demonstration of 4D printing (3D printed object with controlled color changing).

The secondary printing was demonstrated by using the printed prototype of the model of Singapore Stadium as shown in Fig. 3. Using the optimized parameters, the model Singapore Stadium was printed with a yellow color by using the resin of IMRE-PC-G. When the stadium model was exposed to a UV light, the color changed to a dark green. Then an A-Star logo was projected onto the 3D printed model by a projector, and the designed pattern was clearly printed on the 3D printed structures. (Fig. 3c). To further explore the application of the secondary printed technique, the developed secondary printing technique was employed to develop the 3D structures with color gradient or patterns. As shown in Fig. 4, the as printed yellow vase from resin IMRE-PC-G can be easily printed with a gradient color change, or different color patterns. This capability to print secondarily on the 3D structures with different color and pattern is critical important for industry design, providing a fast and cost-saving product design process.
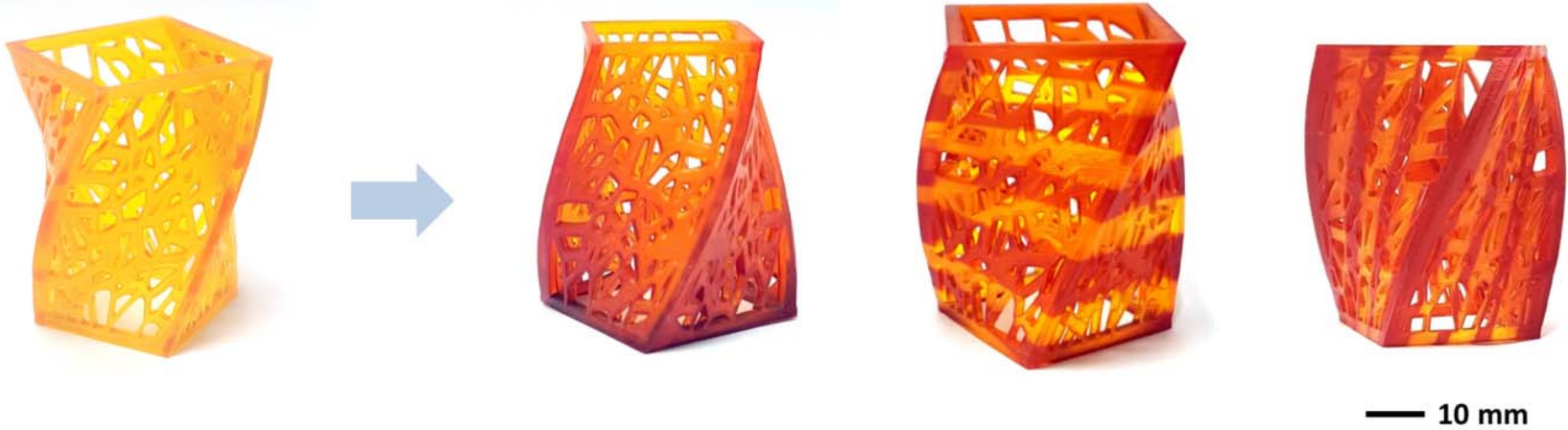

Figure 4. Demonstration of 4D printing: different color patternswith a single 3D printed object.

\section{Summary}

In conclusion, we here reported the first attempt to develop photochromic resins for SLA/DLP based 3D printing technologies. These new photochromic resins were successfully employed in the DLP 3D printing technique, and prototypes with different 3D structures were successfully printed. Most importantly, we demonstrated 3D printed structures with photochromic property can be used for secondary printing, to print additional color gradients or color patterns, which is important for advanced 3D printing technology, especially for industry design. 


\section{Acknowledgement}

We acknowledge financial support of this work from the Science and Engineering Research Council (Grant 1325504107) of Agency for Science, Technology and Research of Singapore.

\section{References}

[1] W. Gao, Y. Zhang, D. Ramanujan, K. Ramani, Y. Chen, C. B. Williams, C. C. L. Wang, Y. C. Shin, S. Zhang and P. D. Zavattieri, Computer-Aided Design 69, 65 (2015).

[2] K. V. Wong and A. Hernandez, ISRN Mechanical Engineering 2012, 10 (2012).

[3] J. W. Stansbury and M. J. Idacavage, Dental Materials 32, 54 (2016).

[4] M. Vaezi, H. Seitz and S. Yang, The International Journal of Advanced Manufacturing Technology 67, 1721 (2013).

[5] P. F. Jacobs, Stereolithography and other RP \& M technologies : from rapid prototyping to rapid tooling, 1996.

[6] S. E. Bakarich, R. Gorkin, M. i. h. Panhuis and G. M. Spinks, Macromolecular Rapid Communications 36, 1211 (2015).

[7] A. Sydney Gladman, E. A. Matsumoto, R. G. Nuzzo, L. Mahadevan and J. A. Lewis, Nat Mater 15, 413 (2016).

[8] A. Lendlein, A. M. Schmidt and R. Langer, Proceedings of the National Academy of Sciences 98, 842 (2001).

[9] M. Zarek, M. Layani, I. Cooperstein, E. Sachyani, D. Cohn and S. Magdassi, Advanced Materials 28, 4449 (2016).

[10] E. Fantino, A. Chiappone, I. Roppolo, D. Manfredi, R. Bongiovanni, C. F. Pirri and F. Calignano, Advanced Materials 28, 3712 (2016).

[11] E. Saleh, P. Woolliams, B. Clarke, A. Gregory, S. Greedy, C. Smartt, R. Wildman, I. Ashcroft, R. Hague, P. Dickens and C. Tuck, Additive Manufacturing 13, 143 (2017).

[12] J. V. Crivello and E. Reichmanis, Chemistry of Materials 26, 533 (2014).

[13] C. Dietlin, S. Schweizer, P. Xiao, J. Zhang, F. Morlet-Savary, B. Graff, J.-P. Fouassier and J. Lalevee, Polymer Chemistry 6, 3895 (2015).

[14] F. Wang, Y. Chong, F. K. Wang and C. He, J. App. Polym. Sci. ASAP, (2017). DOI APP.20170376. 